\title{
Differential induction of malaria liver pathology in mice infected with Plasmodium chabaudi AS or Plasmodium berghei NK65
}

\author{
Diletta Scaccabarozzi ${ }^{1}$, Katrien Deroost ${ }^{2,3}$, Yolanda Corbett ${ }^{1,4}$, Natacha Lays ${ }^{2}$, Paola Corsetto ${ }^{1}$, \\ Fausta Omodeo Salè ${ }^{1 \dagger}$, Philippe E. Van den Steen ${ }^{2 \dagger}$ and Donatella Taramellii ${ }^{* *}$
}

\begin{abstract}
Background: Cerebral malaria and severe anaemia are the most common deadly complications of malaria, and are often associated, both in paediatric and adult patients, with hepatopathy, whose pathogenesis is not well characterized, and sometimes also with acute respiratory distress syndrome (ARDS). Here, two species of murine malaria, the lethal Plasmodium berghei strain NK65 and self-healing Plasmodium chabaudi strain AS which differ in their ability to cause hepatopathy and/or ARDS were used to investigate the lipid alterations, oxidative damage and host immune response during the infection in relation to parasite load and accumulation of parasite products, such as haemozoin.

Methods: Plasma and livers of C57BL/6J mice injected with PbNK65 or PCAS infected erythrocytes were collected at different times and tested for parasitaemia, content of haemozoin and expression of tumour necrosis factor (TNF). Hepatic enzymes, antioxidant defenses and lipids content and composition were also evaluated.

Results: In the livers of P. berghei NK65 infected mice both parasites and haemozoin accumulated to a greater extent than in livers of $P$. chabaudi AS infected mice although in the latter hepatomegaly was more prominent. Hepatic enzymes and TNF were increased in both models. Moreover, in P. berghei NK65 infected mice, increased lipid peroxidation, accumulation of triglycerides, impairment of anti-oxidant enzymes and higher collagen deposition were detected. On the contrary, in P. chabaudi AS infected mice the antioxidant enzymes and the lipid content and composition were normal or even lower than uninfected controls.

Conclusions: This study demonstrates that in C57BL/6J mice, depending on the parasite species, malaria-induced liver pathology results in different manifestations, which may contribute to the different outcomes. In P. berghei NK65 infected mice, which concomitantly develop lethal acute respiratory distress syndrome, the liver tissue is characterized by an excess oxidative stress response and reduced antioxidant defenses while in P. chabaudi AS infected mice hepatopathy does not lead to lipid alterations or reduction of antioxidant enzymes, but rather to inflammation and cytokine burst, as shown earlier, that may favour parasite killing and clearance of the infection. These results may help understanding the different clinical profiles described in human malaria hepatopathy.
\end{abstract}

\section{Background}

Despite large investments and global interventions over the last century, malaria remains a leading cause of

\footnotetext{
*Correspondence: donatella.taramelli@unimi.it

${ }^{\dagger}$ Fausta Omodeo Salè and Philippe E. Van den Steen contributed equally to this work

1 Department of Pharmacological and Molecular Sciences, Università degli Studi di Milano, Milan, Italy

Full list of author information is available at the end of the article
}

morbidity and mortality in humans with 212 million clinical cases in 2015 and 429,000 deaths estimated worldwide, $70 \%$ of which are children under 5 year of age [1]. The malaria infection can occur with fever and generalized sickness, and progress to severe anaemia or cerebral malaria, or can be asymptomatic. This range of clinical courses can be attributed to the host or to the parasites. In particular, the genetic background and the immune system of the host, the dynamics of transmission and 
the virulence of the parasites have to be considered [2, 3]. Hepatic involvement in cases of malarial infection is a well-recognized entity. Malaria hepatocellular dysfunction is characterized by an increase in the serum levels of bilirubin and aminotransferases, exceeding three times the upper limit of normal values. Malarial hepatopathy is associated with a higher incidence of cerebral malaria, shock, acute respiratory distress syndrome (ARDS) and acute kidney injury [4]. It occurs both in adult and paediatric patients, although it is more common in adults $[3,4]$. One suspected cause of malarial hepatopathy is related to the activation of liver macrophages that phagocytize haemozoin or parasitized red blood cells [5]. Focal hepatocyte necrosis, cholestasis and granulomatous lesions have been documented in all malarial nodules [4]. In malaria patients with jaundice, histopathological changes in the form of damaged hepatocytes, congestion of liver cells, haemozoin deposition, inflammatory infiltrates, and cholestasis have been demonstrated [6, 7], as well as hyperplastic Kupffer cells [8].

The molecular basis of different manifestations of malaria using two species of murine Plasmodium, namely Plasmodium berghei strain NK65, and Plasmodium chabaudi strain AS have been recently investigated. Plasmodium berghei NK65-infection results in lethal ARDS [9], whereas $P$. chabaudi AS-infection in C57Bl/6J is selfhealing after an initial peak of parasitaemia and recrudescence, and does not induce lung pathology [10, 11]. Plasmodium berghei NK65 and P. chabaudi AS parasites produce different amounts of haemozoin $(\mathrm{Hz})$, and it has been demonstrated that $\mathrm{Hz}$ is pathogenic in the lungs by inducing pulmonary inflammation [12, 13]. Moreover, in mice infected by $P$. berghei NK65, the development of ARDS is associated with biochemical modifications and lipid alterations of lung tissue and disruption of the molecular organization and lipid composition of alveolar surfactant [12].

Excess $\mathrm{Hz}$ deposition was shown in the livers of $P$. berghei NK65-infected mice compared to P. chabaudi AS mice, while hepatomegaly due to inflammation and cell recruitment was higher in $P$. chabaudi AS mice. Just as in the lungs, liver dysfunction with elevated serum ALT and AST levels and production of both pro- and anti-inflammatory cytokines were associated with the amount of haemozoin present in the livers of $P$. chabaudi AS-infected mice. It was intriguing that inflammation, measured as cell recruitment and cytokine production, was much less pronounced in $P$. berghei NK65 livers that had higher amounts of haemozoin than $P$. chabaudi AS livers. Since haemozoin, due to its haem iron content, is strongly pro-oxidant and immunosuppressive $[14,15]$ the present work aimed at investigating the extent of oxidative damage, the lipid alterations and the antioxidant defences present in the liver tissue of $\mathrm{C} 57 \mathrm{BL} / 6 \mathrm{~J}$ mice infected with $P$. berghei NK65, compared to mice infected with $P$. chabaudi AS.

\section{Methods \\ Reagents}

Unless otherwise specified, all biochemicals were purchased from Sigma (Milan, Italy). Standard fatty acid methylesters were purchased from Alltech (Milan, Italy), silica gel plates (Kieselgel 60) and high performance thin layer chromatography (HPTLC) from Merck (Darmstadt, Germany).

\section{Animals}

The infection of C57BL/6J mice were performed by intraperitoneal injection of $10^{4}$ P. berghei NK65 or P. chabaudi AS infected red blood cells, as described [9, 12]. Three different experiments were performed; 6 or 8 mice/group (control and infected mice) were used for each time point and used at day 8 or 10 after infection. The time intervals and the dose of parasites were chosen based on previous experience. All the data were in line with and confirmed our previous study [16]. Mice were euthanized by intraperitoneal injection of a lethal dose of sodium pentobarbital $(200 \mu \mathrm{l}$ at $60 \mathrm{mg} / \mathrm{ml} \mathrm{Nembutal)}$ at day 8 or 10 post infection and blood was removed by heart puncture and centrifuged to separate plasma and RBC.

\section{Preparation of biological specimens}

Mice were perfused with $0.15 \mathrm{M} \mathrm{NaCl}$ containing $0.2 \mathrm{mM}$ butylhydroxytoluene (BHT) as an antioxidant, and livers were removed, weighted and homogenized in Precellys tubes in six volumes of a solution containing $20 \mathrm{mM}$ Tricine, $250 \mathrm{mM}$ sucrose, $5 \mathrm{mM}$ EDTA, $0.2 \mathrm{mM}$ BHT (pH 7.4) and a protease inhibitor cocktail (Sigma, Milan, Italy).

\section{Haemozoin determination}

The content of $\mathrm{Hz}$ in the perfused livers was analysed by haem-enhanced chemoluminescence determination according to Deroost et al. $[13,17]$. The amount of $\mathrm{Hz}$ ( $\mathrm{pmol} / \mathrm{mg}$ tissue) was multiplied by the total weight of the liver and expressed as pmol or nmol haematin/organ.

\section{Lipid analyses}

The extraction of lipids in the liver was performed according to Folch [18]. Dried lipid extracts were dissolved in chloroform and saved at $-80{ }^{\circ} \mathrm{C}$ until further analysis. The content of phospholipid (PL) phosphorus was determined by the Bartlett procedure [19]. Cholesterol (Cho), triglycerides (TG) and cholesterol esters (ChoE) were quantified by densitometric analysis (Camag Reprostar 3) after separation by HPTLC in hexane/ 
diethyl ether/acetic acid (90:10:1 by vol.). A solution of $10 \% \mathrm{CuSO}_{4}$ in $8 \% \mathrm{H}_{3} \mathrm{PO}_{4}$ was used to visualize the spots. The fatty acid composition of the lipid fractions was analysed by gas liquid chromatography according to Corsetto et al. [20]. The levels of thiobarbituric acid reactive substances were used to determinate the lipid peroxidation, as previously reported [21] and expressed as pmoles of malondialdehyde (MDA).

\section{Antioxidant enzymes and other analyses}

Antioxidant enzymes and total glutathione (GSH + GSSG) were determined in the supernatant of liver homogenates obtained after a centrifugation at $12000 \times g$ for $10 \mathrm{~min}$. Protein concentration was determined by the Bradford assay (Bio-Rad, Hercules, CA) [22]. Catalase (CAT), glutathione reductase (GR) and superoxide dismutase (SOD) activity was determined according to Aebi et al. [23], Pinto et al. [24] and by a assay kit (Cayman Michigan, USA), respectively. Total GSH was determined spectrophotometrically by titration with $5-5^{\prime}$-dithiobis-2 nitro benzoic acid (DTNB) according to Beutler [25]. The collagen content of livers, was determined by measuring hydroxyproline in the tissue homogenates [26]. Liver damage was determined by measuring plasma levels of serum alanine transaminase (ALT) and aspartate transaminase (AST) enzymes, according to manufacturer's protocol (Teco Diagnostics, California, USA).

\section{Quantitative reverse transcription-polymerase chain reaction}

The liver homogenates were used to extract and quantify total RNA and to synthetize cDNA. A quantitative PCR was performed on 25 and $12.5 \mathrm{ng}$ cDNA with primer and probe sets from Applied Biosystems according to Deroost et al. [13]. Data were normalized to $18 \mathrm{~S}$ ribosomal RNA levels.

\section{Statistical analysis}

The data are reported as mean \pm standard deviation (SD). ANOVA test followed by Tukey's multiple comparison test with GraphPad Prism 6.0 (Graphpad Software Inc., La Jolla, CA, USA) were performed to compare the groups and replicates. The differences were considered significant when $\mathrm{p}<0.05$. To eliminate possible artefacts (over- or under-estimation) due to hepatomegaly and hepatic oedema, all the biochemical parameters have been expressed as total content/liver.

\section{Results}

Malaria infection in mice

C57BL/6J mice were infected intraperitoneally with $10^{4}$ infected RBC (either P. berghei NK65 or P. chabaudi AS) and the development of parasitaemia was monitored every day whereas the liver pathology was checked on day 8 and 10 after the start of infection. These time intervals and the parasite dose were chosen on the basis of the indications from previous experiments [9]. Parasitaemia was lower in P. chabaudi AS compared to P. berghei NK65 infected mice at both time points (Fig. 1a), whereas hepatomegaly was evident in P. chabaudi AS, but not in $P$. berghei NK65-infected mice, corroborating previous data [16] (Fig. 1b). Similarly, elevated levels of AST and ALT were detected in the serum of infected mice at both time points (Additional file 1, Panel A and B). Of notice is the observation that in P. berghei NK65 infected mice, AST levels were particularly increased over ALT levels, leading to an AST/ALT ratio of 3.9 and 5.9 at day 8 and 10 , respectively. Differently, in $P$. chabaudi AS infected mice the AST/ALT ratios was 1.5 and 1.2 at the same time intervals (Additional file 1, Panel C). These findings are consistent with the levels of parasitaemia in P. berghei NK65 mice since elevated AST levels may also reflect haemolysis in addition to liver damage [27]. Previous
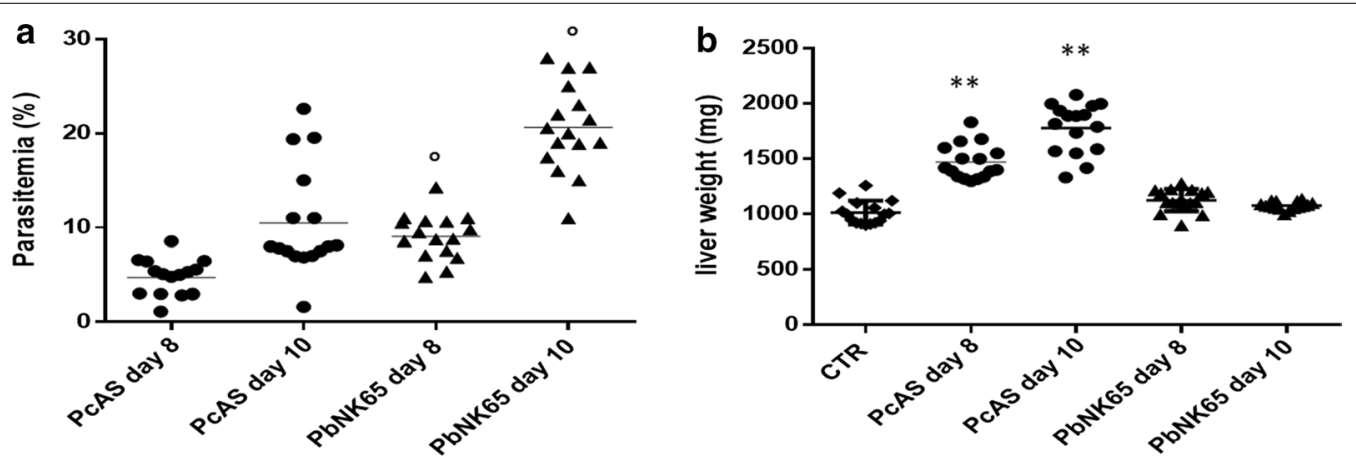

Fig. 1 Disease course in mice infected with P. berghei NK65 or P. chabaudi AS. C57BL/6J mice were injected intraperitoneally with $10^{4}$ erythrocytes infected with P. berghei (PbNK65) or P. chabaudi AS (PCAS). Peripheral parasitaemia (a) and weights of the livers (b) were determined at day 8 and 10 post infection. $n=15-16$ mice for each time point and strain. Data are pooled from three different experiments performed in the same conditions. ${ }^{* *} p<0.01$; vs control; ${ }^{\circ} p<0.05$ P. berghei NK65 vs P. chabaudi AS 
reports in a larger set of experiments confirm these observations. [16].

Differently from $P$. chabaudi AS-infected mice, livers from $P$. berghei NK65-infected mice also showed a darkbrown coloration in agreement with the significantly higher hepatic $\mathrm{Hz}$ deposition (Fig. 2a). In parallel with these modifications, also the proinflammatory status of the liver changed during the infections with the different Plasmodium species. The mRNA levels of TNF increased during the progression of the disease in the livers of both $P$. berghei NK65 and $P$. chabaudi AS infected mice (Fig. 2b), but with a different kinetic and values. Collagen deposition, measured as hydroxyproline content, was significantly higher with both parasite species at day 8 post infection with the highest levels in P. berghei NK65 livers. At day 10, the values returned to normal in both groups of mice, as those of control (Fig. 2c). Lipid peroxidation, expressed as total pmoles MDA/liver, was found to be significantly and time-dependently increased in mice infected with both Plasmodium species showing higher levels in P. berghei NK65 infected mice at either day post infection (Fig. 2d).

\section{Oxidative stress response in the liver}

The above findings, namely the increased levels of $\mathrm{Hz}$ and increased levels of MDA suggestive of oxidative stress in $P$. berghei NK65-livers, prompted us to investigate the status of the antioxidant defence systems in the infected livers as a determinant of tissue susceptibility to oxidative damage. The pattern of response in the two groups of infected mice was again different. As expected from the higher levels of lipoperoxidation, the liver extracts of $P$. berghei NK65 infected mice were characterized by a lower CAT activity and lower levels of total glutathione (GSH + GSSG) at both days post infection (Fig. 3a, b). SOD activity was significantly enhanced at day 10 post infection, whereas GR was not different from uninfected mice (Fig. 3c, d). On the contrary, P. chabaudi AS-infected mice showed CAT and SOD activity and GSH levels similar to control at both days post infection, whereas GR activity was higher at both time points (Fig. 3d).

\section{Species-specific lipid changes in infected livers}

Liver dysfunction is generally associated with lipid alterations. The lipid profile of infected livers was significantly
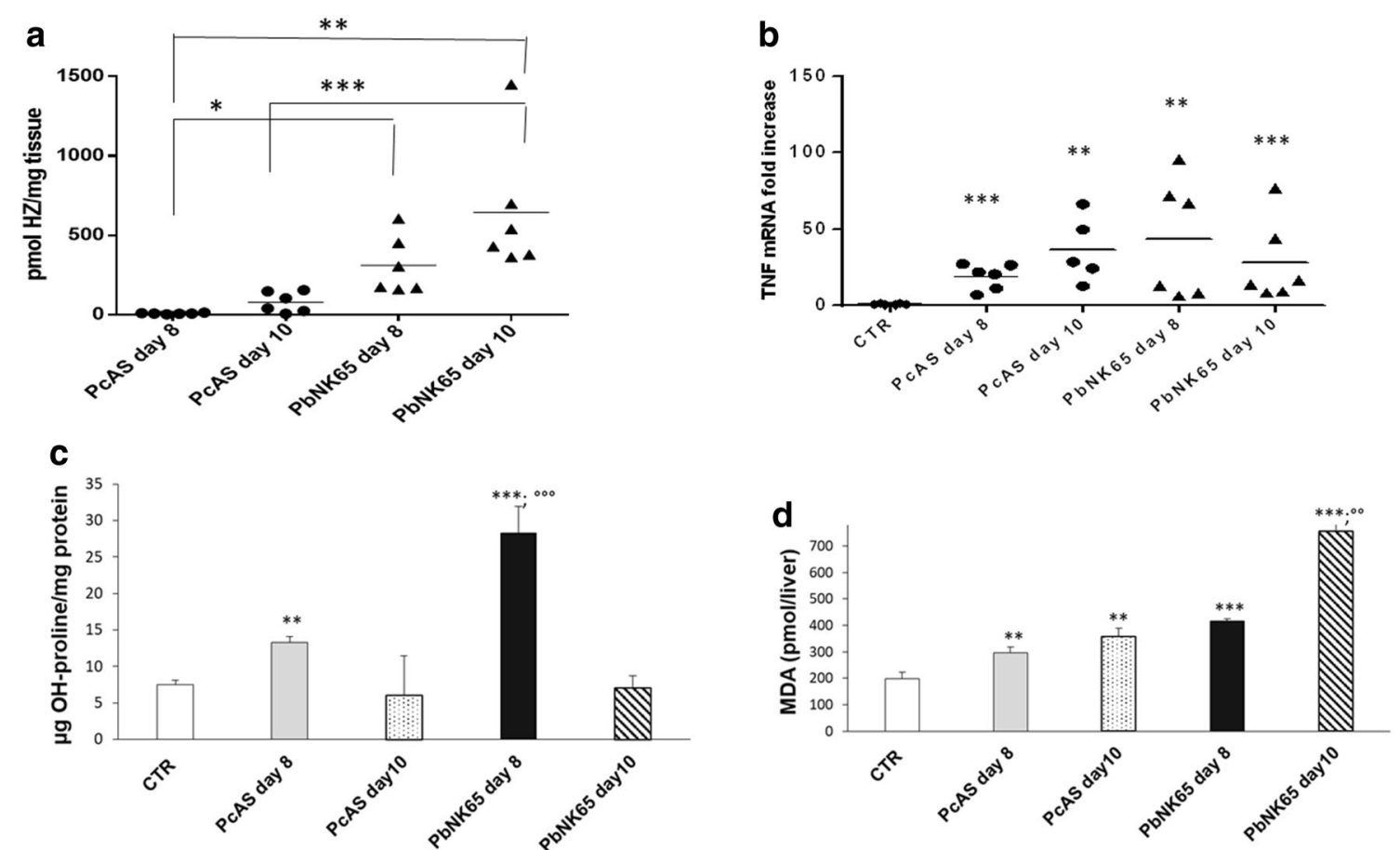

Fig. 2 Hepatic haemozoin levels, oxidative damage and fibrosis in liver tissue of P. berghei NK65 or P. chabaudi AS infected mice. Hz content in liver tissue (pmol Hz/mg liver tissue) of P. berghei NK65 or P. chabaudi AS infected C57BI/6J mice at day 8 and 10 post infection (a). Hepatic mRNA expression levels of TNF-a in non-infected and infected mice at day 8 or day 10 post infection determined by quantitative RT-PCR and normalized to $18 \mathrm{~S}$ ribosomal RNA levels (b). OH-proline content $(\mu \mathrm{g} / \mathrm{mg}$ protein) in non-infected and infected mice at day 8 or day 10 post infection (c); MDA content (pmol/liver) in non-infected and infected mice at day 8 or day 10 post infection (d). $n=6$ mice/for each time point and group. ${ }^{*} p<0.05 ;{ }^{* *} p<0.01$; ${ }^{* * *} \mathrm{p}<0.0001$ vs Control; ${ }^{\circ} \mathrm{p}<0.05 ;{ }^{\circ 00} \mathrm{p}<0.01$ P. berghei NK65 vs P. chabaudi AS 

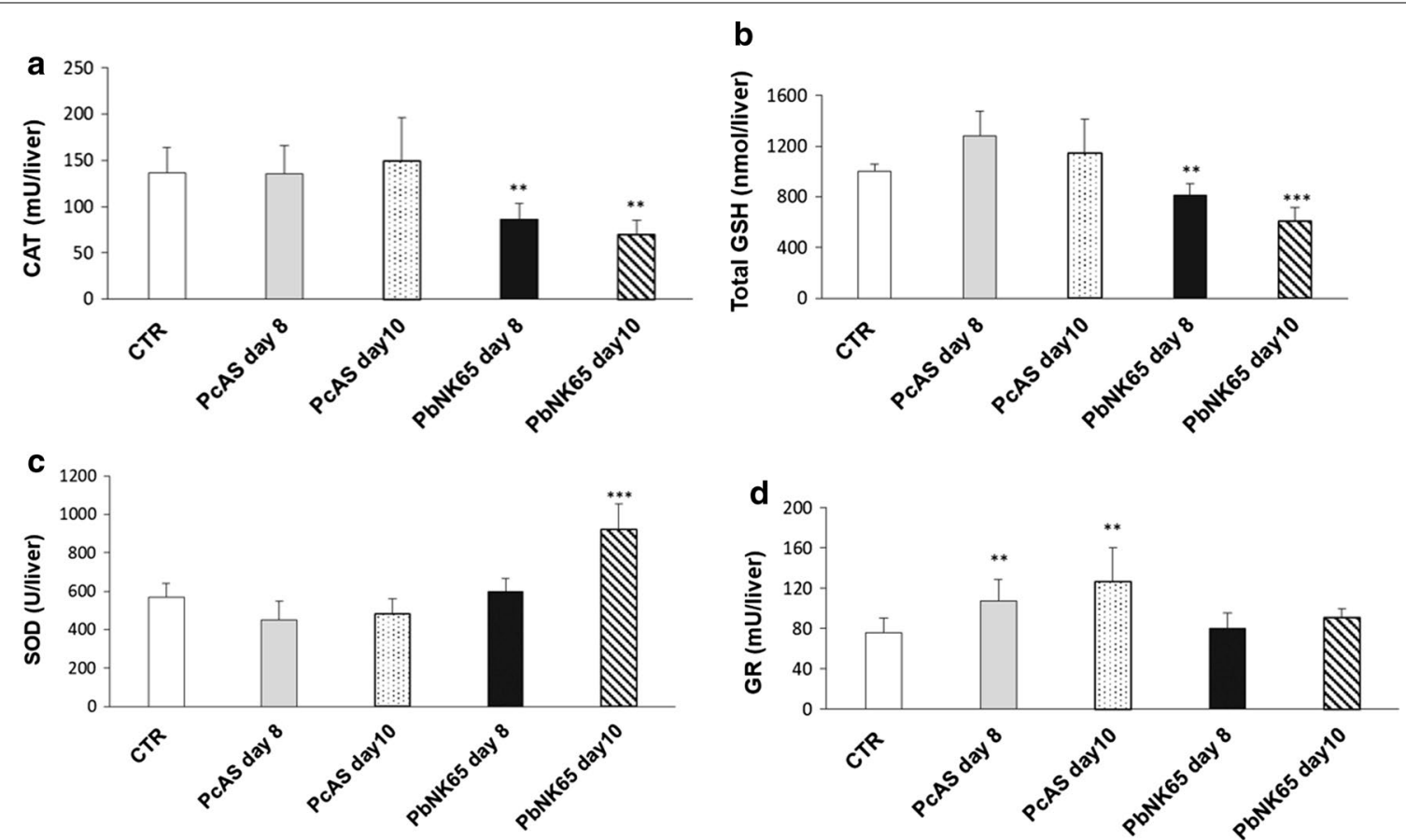

Fig. 3 Enzymes activity in the liver of C57BI/6J mice infected by P. berghei NK65 or P. chabaudi AS. Catalase activity (a), total GSH (b), SOD activity (c) and glutathione reductase activity (d) were determined in the livers of non-infected and infected mice at day 8 and day 10 post infection. $n=8$ mice/group. Data are from one representative experiment out of three performed in the same conditions; ${ }^{*} p<0.05 ;{ }^{* *} p<0.01 ;{ }^{* * *} p<0.0001 \mathrm{vs}$ control

modified compared to control mice and different between the two species. TG levels were significantly higher in the P. berghei NK65- compared to P. chabaudi AS-infected livers (Fig. 4a). In addition, higher levels of ChoE, and lower levels of free Cho were seen with $P b N K 65$ at both day 8 and 10 post infection, compared to control mice, whereas $P$. chabaudi AS-infected mice had higher levels of free cholesterol (Fig. 4b, c). Compared to control mice, PL were more strongly increased in P. chabaudi ASinfected livers than in P. berghei NK65 at both days post infection (Fig. 4d), whereas no differences were found in the composition of the PLs due to infection (Fig. 5).

Modification in the fatty acid distribution between $P$. berghei NK65 vs P. chabaudi AS livers was also observed. Palmitic acid was significantly increased in P. chabaudi AS livers compared to control, whereas this was decreased in P. berghei NK65 together with palmitoleic acid. Among the polyunsaturated fatty acids, significantly higher amounts of linoleic acid $(\mathrm{C} 18: 2 \mathrm{n}-6)$ and lower levels of eicosatrienoic $(\mathrm{C} 20: 3 \mathrm{n}-6)$ and arachidonic (C20:4 n - 6) acids were seen in P. berghei NK65 compared to control (Table 1). These differences were less pronounced in $P$. chabaudi AS livers and limited to the changes of arachidonic acid (C20:4 n - 6) and docosapentaenoic acid (C22:5 n-3). As a consequence of these changes, also the $\mathrm{C} 18: 2 / \mathrm{C} 20: 4$ ratio was significantly increased in $P$. berghei NK65 compared to control or $P$. chabaudi AS.

\section{Discussion}

The pathogenesis of hepatic dysfunction in malaria is complex and not completely understood. Histopathological findings show congested and swollen hepatocytes, Kupffer cell hyperplasia, deposition of brown malarial pigment, mononuclear cell infiltration, micro-occlusion by parasitized RBC and, less frequently, steatosis and spotty and submassive necrosis $[2,7,13,16,28]$. Infection, inflammation and oxidative stress have been shown to induce multiple alterations in hepatic lipid and lipoprotein metabolism [29]. The present study demonstrates that blood stage malaria infection by $P$. chabaudi AS or P. berghei NK65 exerts different effects on the liver of C57BL/6J mice. Hepatomegaly was confirmed in P. chabaudi AS mice, as previously shown [16] as well as liver dysfunction suggested by the serum increase of the hepatic enzymes AST and ALT, especially at day 10 post infection. The high AST levels lead to an AST/ALT ratio significantly higher in $P$. berghei NK65 compared to $P$. chabaudi AS. The AST/ALT index is used to discriminate the type of liver damage observed $[27,30]$ and a progressive increase in the AST/ALT ratio correlated with a decrease in liver functions, like in cirrhosis and fibrosis 

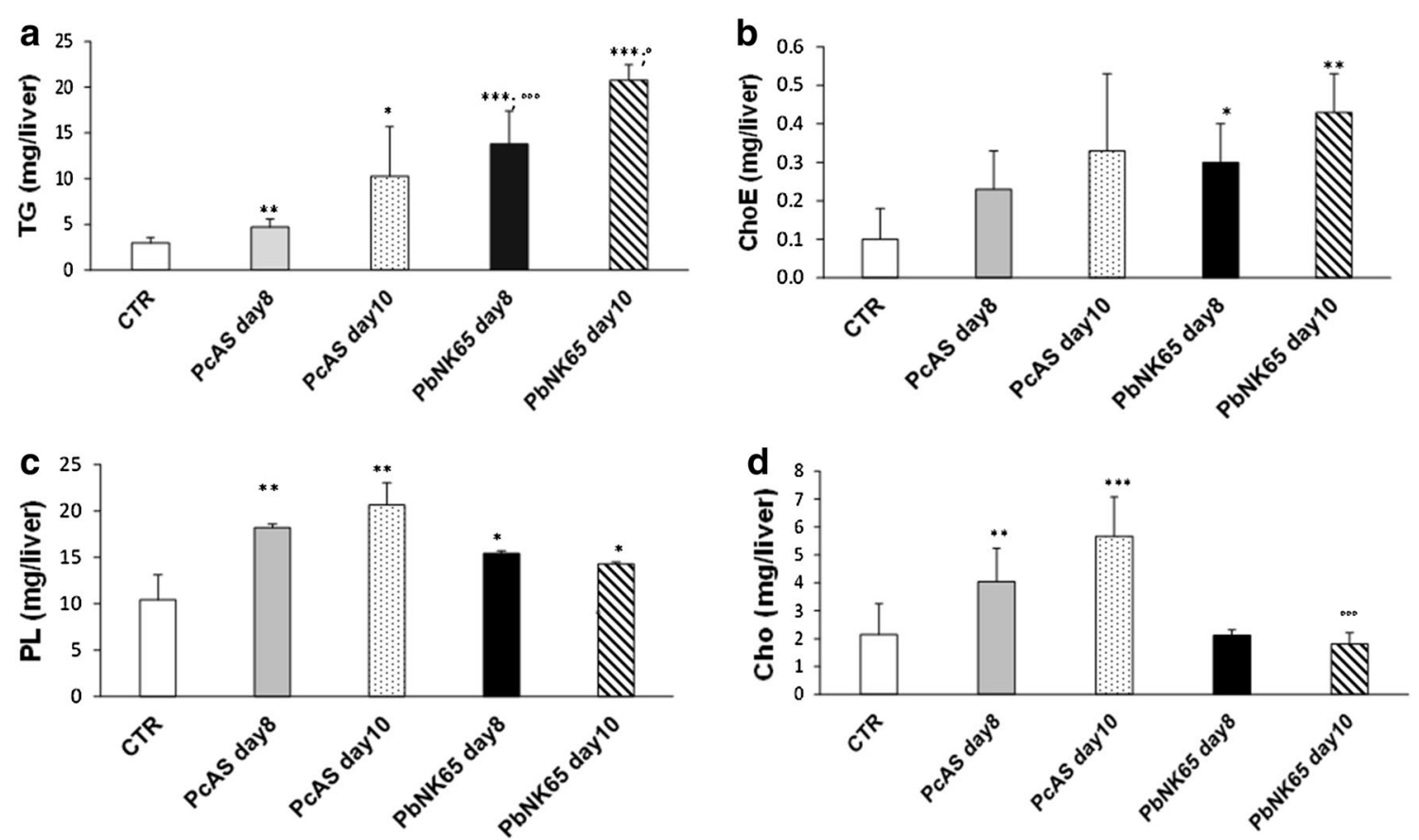

Fig. 4 Alteration in the lipid profiles of the livers from uninfected, P. berghei NK65 or P. chabaudi AS infected mice. Mice infected with P. berghei NK65 or P. chabaudi AS were dissected at day 8 and 10 post infection. The total content of liver triglycerides (TG) and phospholipids (PL) were determined as described in "Methods" (a and $\mathbf{c}$ ). The content of ChoE and free Cho is shown in $\mathbf{b}$ and $\mathbf{d}$, respectively. $\mathrm{n}=8$ mice for each condition. Data are from one representative experiment out of three performed in the same conditions ${ }^{*} p<0.05 ;{ }^{* *} p<0.01 ;{ }^{* * *} p<0.0001$ vs Control; ${ }^{\circ} p<0.05$; ${ }^{000} \mathrm{p}<0.001$ P. berghei NK65 vs P. chabaudi AS

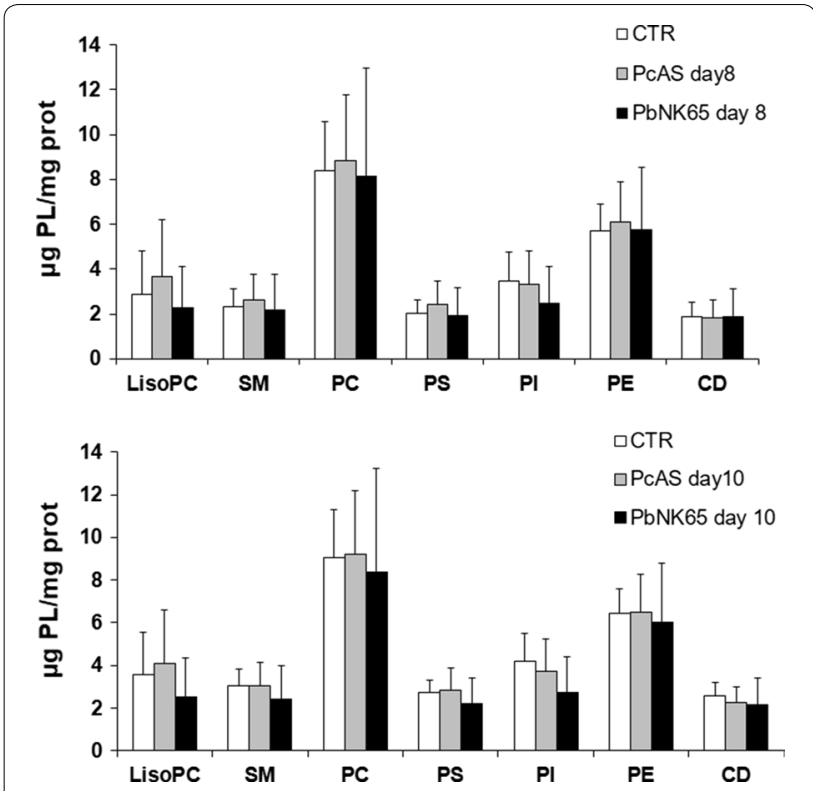

Fig. 5 Phospholipids composition in liver from uninfected, P. berghei NK65 or P. chabaudi AS infected mice. The distribution of liver PL ( $\mu \mathrm{g}$ $\mathrm{PL} / \mathrm{mg}$ protein) was determined in uninfected and in P. berghei NK65or P. chabaudi AS-infected mice at day 8 or 10 post infection. Data are from one representative experiment out of three performed in the same conditions. LPC lysophosphatidylcholine, SM sphingomyelin, $P C$ phosphatidylcholine, PS phosphatidylserine, P/ phosphatidylinositol, $P E$ phosphatidylethanolamine, $C D$ cardiolipine
[31]. Since plasma clearance of AST is modulated by the activity of sinusoidal liver cells, during progressive fibrosis and cirrhosis, the functions of these cells are progressively impaired resulting in a relative increase in AST levels [32]. In the case of malaria, in P. berghei NK65infected mice, an alternative hypothesis can be proposed. Elevated AST levels in absence of elevated ALT, in fact, may indicate haemolysis, since RBC contain AST. This has already been reported in sickle cell disease where the AST/ALT ratio has been used as haemolytic index [33]. In the models used for this work, the AST/ALT ratio may well indicate initial fibrosis in both species, as confirmed by the $\mathrm{OH}$-proline increase at day 8 post infection, and increased haemolysis in $P$. berghei NK65-infected mice that develop higher parasitaemia than $P$. chabaudi AS mice. The latter leads to higher levels of $\mathrm{Hz}$ in the liver of $P$. berghei NK65- than in P. chabaudi AS-infected mice [16]. It is well known that $\mathrm{Hz}$ plays a crucial role in generating oxidative damage $[12,14,15]$. The higher content of $\mathrm{Hz}$, free (pro-oxidant) haem derived from intravascular haemolysis and high parasitaemia are likely to contribute to excessive oxidative stress response in $P$. berghei NK65 livers. TNF may also participate. A marginal role seems to be played by the inflammatory infiltrate, which is much less abundant in $P$. berghei NK65-infected livers than in $P$. chabaudi AS mice, in which increased liver 
Table 1 Fatty acid distribution in the liver tissue of Plasmodium chabaudi AS or Plasmodium berghei NK65 infected mice at different days after the infection

\begin{tabular}{|c|c|c|c|c|c|}
\hline & \multicolumn{5}{|l|}{$\%$ of total } \\
\hline & Control & $\begin{array}{l}\text { P. chabaudi } \\
\text { AS day } 8\end{array}$ & $\begin{array}{l}\text { P. chabaudi } \\
\text { AS day } 10\end{array}$ & $\begin{array}{l}\text { P. berghei } \\
\text { NK65 day } 8\end{array}$ & $\begin{array}{l}\text { P. berghei } \\
\text { NK65 day } 10\end{array}$ \\
\hline C16:0 palmitic acid & $20.76 \pm 1.0$ & $25.04 \pm 0.8^{*}$ & $26.10 \pm 0.1^{*}$ & $18.41 \pm 0.9^{*}$ & $18.01 \pm 0.2^{*}$ \\
\hline C16:1 palmitoleic acid & $1.10 \pm 0.2$ & $1.24 \pm 0.1$ & $1.17 \pm 0.1$ & $0.63 \pm 0.3^{*}$ & $0.59 \pm 0.1^{*}$ \\
\hline C18:0 stearic acid & $13.25 \pm 1.6$ & $12.80 \pm 0.7$ & $13.47 \pm 1.1$ & $13.54 \pm 1.7$ & $13.87 \pm 0.9$ \\
\hline C18:1 oleic acid & $10.12 \pm 1.5$ & $9.98 \pm 1.5$ & $11.01 \pm 1.2$ & $10.52 \pm 1.5$ & $10.20 \pm 1.0$ \\
\hline C18:2 n - 6 linoleic acid & $15.00 \pm 1.4$ & $17.4 \pm 0.6$ & $17.98 \pm 1.2$ & $22.19 \pm 2.4^{* *}$ & $23.01 \pm 1.9^{* *}$ \\
\hline C18:3 n - 3 a-linolenic acid & $0.25 \pm 0.1$ & $0.22 \pm 0.1$ & $0.19 \pm 0.1$ & $0.21 \pm 0.1$ & $0.20 \pm 0.1$ \\
\hline C18:3 n - 6 p-linolenic acid & $0.17 \pm 0.1$ & $0.18 \pm 0.1$ & $0.17 \pm 0.1$ & $0.16 \pm 0.1$ & $0.19 \pm 0.1$ \\
\hline c20:3 n - 6 eicosatrienoic acid & $1.20 \pm 0.1$ & $0.98 \pm 0.2$ & $1.10 \pm 0.1$ & $0.51 \pm 0.1^{* * *}$ & $0.49 \pm 0.1^{* * *}$ \\
\hline C20:4 n - 6 arachidonic acid & $22.02 \pm 1.1$ & $19.99 \pm 0.8^{*}$ & $18.97 \pm 1.0^{*}$ & $16.81 \pm 1.4^{* * *}$ & $16.01 \pm 1.2^{* * *}$ \\
\hline c20:5 n - 3 eicosapentaenoic acid & $0.29 \pm 0.1$ & $0.22 \pm 0.1$ & $0.30 \pm 0.1$ & $0.21 \pm 0.1$ & $0.29 \pm 0.1$ \\
\hline c22:5 n - 3 docosapentaenoic acid & $0.38 \pm 0.1$ & $0.57 \pm 0.1^{*}$ & $0.60 \pm 0.2^{*}$ & $0.37 \pm 0.1$ & $0.39 \pm 0.1$ \\
\hline C22:6 n - 3 docosahexanoic acid & $9.28 \pm 0.7$ & $9.26 \pm 1.2$ & $9.40 \pm 1.0$ & $9.79 \pm 0.6$ & $9.70 \pm 0.9$ \\
\hline C18:2/C20:4 & $0.68 \pm 0.1$ & $0.87 \pm 0.1$ & $0.95 \pm 0.1^{*}$ & $1.32 \pm 0.1^{* *}$ & $1.44 \pm 0.2^{* * *}$ \\
\hline
\end{tabular}

Data are expressed as mean $\pm S D$

${ }^{*} \mathrm{p}<0.05 ;{ }^{* *} \mathrm{p}<0.01$; *** $\mathrm{p}<0.001$ vs Control $\mathrm{n}=6-7$

weight, histology and the correlation between hepatic inflammation, enzymes and haemozoin were demonstrated [16].

The higher production of ROS in P. berghei NK65infected mice was confirmed by the elevated concentration of MDA, which is an index for the loss of structure and cell membrane integrity, and the concomitant depletion of the antioxidant defence system, such as CAT and total GSH. GSH constitutes the first line of defence against free radicals and is a critical determinant of tissue susceptibility to oxidative damage. The increase of SOD, shown also in other models of liver damage [34], reveals the necessity of minimizing oxidative stress, which largely derives from the production of superoxide anion radicals. These changes seems to be associated with $P$. berghei NK65 infection, since they were not present in $P$. chabaudi AS-infected mice that showed only an increase of GR at both days post infection.

An important change observed in P. berghei NK65 vs $P$. chabaudi AS mice was the augmentation of the liver content of TG and cholesterol esters that indicates a potent stimulation of hepatic lipogenesis by $P$. berghei parasites [35]. This is in agreement with literature data showing that livers from $P$. berghei-infected mice contain lipid droplets and myelin-like figures [36]. A significant, although limited increase of TG was also present in $P$. chabaudi AS infected mice in agreement to the small neutral lipid inclusions observed by Seixas et al. [37]. Elevated TG are a characteristic of liver pathologies of different aetiology, clinically defined as non-alcoholic fatty liver disease in which TG accumulate and plasma albumin and protein decrease.

On the contrary, higher amounts of PL and free Cho were present in $P$. chabaudi AS-infected mice compared to contr or $P$. berghei NK65-infected mice. This is possibly related to reticuloendothelial hyperplasia and/or cholestasis, similar to those reported in the same or other models of malaria infection [7,38]. The fatty acid distribution in the liver tissue of $P$. chabaudi AS or P. berghei NK65 infected mice at different days after the infection confirms the impairment (possibly induced by ROS) of the elongation/desaturation pathway from linoleic to arachidonic acid and may explain the higher linoleic/arachidonic acid ratio in the liver of $P$. berghei NK65-infected mice [39]. These observations also contribute to explain the changes in the lipid content and composition that was reported in the lungs of $P$. berghei NK65 infected mice [12].

All together, these findings are in agreement with other models of $P$. berghei infection and support the hypothesis that ROS may play a critical role in the liver dysfunction caused by $P$. berghei NK65 parasites $[35,40]$. Consistent with this hypothesis are also the signs of fibrosis, revealed by the elevated hepatic levels of hydroxyproline at day 8 post infection in P. berghei NK65-infected mice. Indeed, it is well known that oxidative stress is one of the major stimuli of fibrosis [41]. Excess of ROS may induce severe liver damage followed by a phase of repair [42] during which TGF- $\beta 1$ on one hand limits the proliferative response of hepatocytes, and on the other hand, increases 
the production of collagen and other extracellular matrix proteins. Submicroscopical signs of fibrosis were evident also in P. chabaudi AS-infected mice, although at a lower extent. Interestingly, at day 10 post infection, the amount of hydroxyproline decreased with both parasite species, becoming similar to control mice. The hypothesis that inflammation may lead to the activation of matrix metalloproteinases (MMPs) is under investigation. MMPs are proteolytic enzymes able to degrade different proteins of the extracellular matrix (e.g. collagen, laminin, fibronectin) and modulate cytokine and chemokine activity in cases of severe inflammation. Previous data indicated that, in the liver of $\mathrm{C} 57 \mathrm{Bl} / 6 \mathrm{~J}$ mice infected with $P$. berghei ANKA, the activity of MMPs is significantly increased [43]. Furthermore, several in vitro studies have shown a TNF-dependent induction of MMPs at both mRNA and protein levels in monocytes fed with natural $\mathrm{Hz}[16,44]$.

\section{Conclusion}

In conclusion, this study demonstrates that, in C57BL/6J mice, $P$. berghei NK65 and $P$. chabaudi AS parasite species induce liver pathology in a different manner. In particular, in P. berghei NK65 infected mice, which succumb of malaria ARDS, it seems that an excess oxidative response predominates, as demonstrated by the alteration of the liver anti-oxidant enzymes, the increased MDA levels and the accumulation of TG. These changes are related to the high parasitaemia, the liver $\mathrm{Hz}$ deposition and the modification in protein content and lipid profile. These alterations are less pronounced with $P$. chabaudi AS, which develops a self-limiting infection, with lower parasitaemia and $\mathrm{Hz}$ deposition, and thus less oxidative stress. The cell infiltrate and the cytokine burst occurred at the peak of the infection may suggest an inflammatory liver response, which precedes parasite clearance [16].

\section{Additional file}

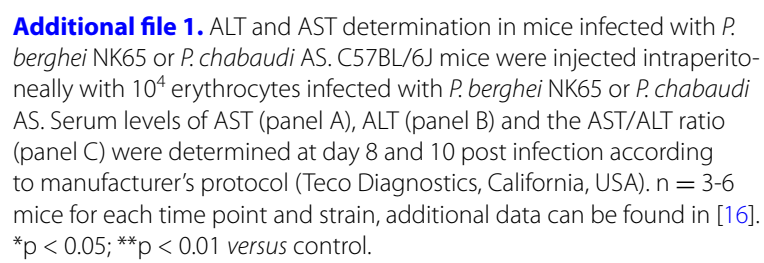

\footnotetext{
Abbreviations

ALT: alanine transaminase; ARDS: acute respiratory distress syndrome; AST: aspartate transaminase; BHT: butylhydroxytoluene; CAT: catalase; Cho: cholesterol; ChoE: cholesterol esters; GR: glutathione reductase; GSH + GSSG: total glutathione; HPTLC: high performance thin layer chromatography; $\mathrm{Hz}$ : haemozoin; MDA: malondialdehyde; PbNK65: Plasmodium berghei NK65; PCAS: Plasmodium chabaudi AS; PL: phospholipid; SOD: superoxide dismutase; TG: triglycerides; TNF: tumor necrosis factor.
}

\section{Authors' contributions}

DS: Data collection, data analysis and interpretation, drafting the article. KD: Data collection and data interpretation. YC, PC and NL: Data collection. PVDS: Data analysis and interpretation, critical revision of the article, final approval of the version to be published. FOS: Data analysis and interpretation, critical revision of the article. DT: Data interpretation, critical revision of the article, final approval of the version to be published. All authors read and approved the final manuscript.

\section{Author details}

${ }^{1}$ Department of Pharmacological and Molecular Sciences, Università degli Studi di Milano, Milan, Italy. ${ }^{2}$ Rega Institute for Medical Research, KU LeuvenUniversity of Leuven, Louvain, Belgium. ${ }^{3}$ Present Address: The Francis Crick Institute, London, UK. ${ }^{4}$ Present Address: Dipartimento di Bioscienze, Università degli Studi di Milano, Milan, Italy.

\section{Acknowledgements}

The excellent technical help of Gigliola Montorfano is acknowledged.

\section{Competing interests}

The authors declare that they have no competing interests.

Availability of data and materials

Not applicable.

\section{Consent for publication}

Not applicable.

\section{Ethics approval and consent to participate}

All experiments were approved by the Animal Ethics Committee from the KU Leuven (License LA1210186, Belgium). All efforts were made to minimize animal suffering

\section{Funding}

This work has been supported by the Foundation for Scientific Research of Flanders (FWO-Vlaanderen) and the Geconcerteerde Onderzoeksacties (GOA 2013/014 and C1 2017-2023) of the research fund of the KU Leuven-University of Leuven. PVDS is a research professor at the KU Leuven. Additional support comes from the MIUR-PRIN project 20154JRJPP_004 to DT.

\section{Publisher's Note}

Springer Nature remains neutral with regard to jurisdictional claims in published maps and institutional affiliations.

Received: 20 September 2017 Accepted: 23 December 2017

Published online: 09 January 2018

\section{References}

1. WHO. Malaria report. Geneva: World Health Organization; 2017.

2. White NJ, Pukrittayakamee S, Hien TT, Faiz MA, Mokuolu OA, Dondorp AM. Malaria. Lancet. 2014;383:723-35.

3. Wassmer SC, Taylor TE, Rathod PK, Mishra SK, Mohanty S, ArevaloHerrera M, et al. Investigating the pathogenesis of severe malaria: a multidisciplinary and cross-geographical approach. Am J Trop Med Hyg. 2015;93:42-56.

4. Jain A, Kaushik R, Kaushik RM. Malarial hepatopathy: clinical profile and association with other malarial complications. Acta Trop. 2016;159:95-105.

5. Anand AC, Puri P. Jaundice in malaria. J Gastroenterol Hepatol. 2005;20:1322-32

6. Wilairatana P, Looareesuwan S, Charoenlarp P. Liver profile changes and complications in jaundiced patients with falciparum malaria. Trop Med Parasitol. 1994;45:298-302.

7. Kochar DK, Agarwal P, Kochar SK, Jain R, Rawat N, Pokharna RK, et al. Hepatocyte dysfunction and hepatic encephalopathy in Plasmodium falciparum malaria. QJM. 2003;96:505-12. 
8. Viriyavejakul P, Khachonsaksumet V, Punsawad C. Liver changes in severe Plasmodium falciparum malaria: histopathology, apoptosis and nuclear factor kappa B expression. Malar J. 2014;13:106.

9. Van den Steen PE, Geurts N, Deroost K, Van Aelst I, Verhenne S, Heremans $\mathrm{H}$, et al. Immunopathology and dexamethasone therapy in a new model for malaria-associated acute respiratory distress syndrome. Am J Respir Crit Care Med. 2010;181:957-68.

10. Stevenson MM, Lyanga JJ, Skamene E. Murine malaria: genetic control of resistance to Plasmodium chabaudi. Infect Immun. 1982;38:80-8.

11. Geurts N, Martens E, Verhenne S, Lays N, Thijs G, Magez S, et al. Insufficiently defined genetic background confounds phenotypes in transgenic studies as exemplified by malaria infection in TIr9 knockout mice. PLoS ONE. 2011;6:e27131.

12. Scaccabarozzi $D$, Deroost $K$, Lays N, Omodeo Salè F, Van den Steen PE, Taramelli D. Altered lipid composition of surfactant and lung tissue in murine experimental malaria-associated acute respiratory distress syndrome. PLoS ONE. 2015;10:e0143195.

13. Deroost K, Tyberghein A, Lays N, Noppen S, Schwarzer E, Vanstreels E, et al. Hemozoin induces lung inflammation and correlates with malariaassociated acute respiratory distress syndrome. Am J Respir Cell Mol Biol. 2013;48:589-600.

14. Taramelli D, Recalcati S, Basilico N, Olliaro P, Cairo G. Macrophage preconditioning with synthetic malaria pigment reduces cytokine production via heme iron-dependent oxidative stress. Lab Invest. 2000;80:1781-8.

15. Schwarzer E, Arese P, Skorokhod OA. Role of the lipoperoxidation product 4-hydroxynonenal in the pathogenesis of severe malaria anemia and malaria immunodepression. Oxid Med Cell Longev. 2015;2015:638416.

16. Deroost K, Lays N, Pham TT, Baci D, Van den Eynde K, Komuta M, et al. Hemozoin induces hepatic inflammation in mice and is differentially associated with liver pathology depending on the Plasmodium strain. PLoS ONE. 2014;9:e113519.

17. Deroost K, Lays N, Noppen S, Martens E, Opdenakker G, Van den Steen PE. Improved methods for haemozoin quantification in tissues yield organ-and parasite-specific information in malaria-infected mice. Malar J. 2012;11:166.

18. Folch J, Lees M, Sloane Stanley GH. A simple method for the isolation and purification of total lipides from animal tissues. J Biol Chem. 1957:226:497-509.

19. Bartlett GR. Phosphorus assay in column chromatography. J Biol Chem. 1959;234:466-8.

20. Corsetto PA, Montorfano G, Zava S, Jovenitti IE, Cremona A, Berra B, et al. Effects of n-3 PUFAs on breast cancer cells through their incorporation in plasma membrane. Lipids Health Dis. 2011;10:73.

21. D'Alessandro S, Basilico N, Corbett Y, Scaccabarozzi D, Omodeo-Salè F, Saresella M, et al. Hypoxia modulates the effect of dihydroartemisinin on endothelial cells. Biochem Pharmacol. 2011;82:476-84.

22. Bradford MM. A rapid and sensitive method for the quantitation of microgram quantities of protein utilizing the principle of protein-dye binding. Anal Biochem. 1976;72:248-54.

23. Aebi H. Catalase in vitro. Methods Enzymol. 1984;105:121-6.

24. Pinto MC, Mata AM, Lopez-Barea J. Reversible inactivation of Saccharomyces cerevisiae glutathione reductase under reducing conditions. Arch Biochem Biophys. 1984;228:1-12.

25. Beutler E. Red cell metabolism — a manual of Biochemical Methods. 3rd ed. Orlando: Grune and Stratton; 1984

26. Tager AM, Kradin RL, LaCamera P, Bercury SD, Campanella GS, Leary CP, et al. Inhibition of pulmonary fibrosis by the chemokine IP-10/CXCL10. Am J Respir Cell Mol Biol. 2004;31:395-404.
27. Kumar D, Sharma CB. A comparative study of serum Alt \& Ast level and Ast/Alt ratio in alcoholic and non-alcoholic acute uncomplicated falciparum malaria without clinical jaundice. J Dental Med Sci. 2017;16:6-8.

28. Kochar DK, Singh P, Agarwal P, Kochar SK, Pokharna R, Sareen PK. Malarial hepatitis. J Assoc Phys India. 2003;51:1069-72.

29. Khovidhunkit W, Kim MS, Memon RA, Shigenaga JK, Moser AH, Feingold $\mathrm{KR}$, et al. Effects of infection and inflammation on lipid and lipoprotein metabolism: mechanisms and consequences to the host. J Lipid Res. 2004;45:1169-96.

30. Gowda S, Desai PB, Hull VV, Math AA, Vernekar SN, Kulkarni SS. A review on laboratory liver function tests. Pan Afr Med J. 2009;3:17.

31. Giannini E, Botta F, Fasoli A, Ceppa P, Risso D, Lantieri PB, et al. Progressive liver functional impairment is associated with an increase in AST/ALT ratio. Dig Dis Sci. 1999;44:1249-53.

32. Giannini EG, Zaman A, Ceppa P, Mastracci L, Risso D, Testa R. A simple approach to noninvasively identifying significant fibrosis in chronic hepatitis C patients in clinical practice. J Clin Gastroenterol. 2006;40:521-7.

33. Nsiah K, Dzogbefia VP, Ansong D, Osei Akoto A, Boateng H, Ocloo D. Pattern of AST and ALT changes in relation to hemolysis in sickle cell disease. Clin Med Insights Blood Disord. 2011:4:1-9.

34. Salatti Ferrari R, Pase da Rosa D, Forgiarini LF, Bona S, Dias Simoes A, Possa Marroni N. Oxidative stress and pulmonary changes in experimental liver cirrhosis. Oxid Med Cell Longev. 2012;2012:486190.

35. Akanbi OM, Omonkhua AA, Cyril-Olutayo CM, Fasimoye RY. The antiplasmodial activity of Anogeissus leiocarpus and its effect on oxidative stress and lipid profile in mice infected with Plasmodium berghei. Parasitol Res. 2012:110:219-26.

36. Rodriguez-Acosta A, Finol HJ, Pulido-Mendez M, Marquez A, Andrade G, Gonzalez N, et al. Liver ultrastructural pathology in mice infected with Plasmodium berghei. J Submicrosc Cytol Pathol. 1998;30:299-307.

37. Seixas E, Oliveira P, Moura Nunes JF, Coutinho A. An experimental model for fatal malaria due to TNF-alpha-dependent hepatic damage. Parasitology. 2008;135:683-90.

38. Brugat T, Cunningham D, Sodenkamp J, Coomes S, Wilson M, Spence PJ, et al. Sequestration and histopathology in Plasmodium chabaudi malaria are influenced by the immune response in an organ-specific manner. Cell Microbiol. 2014;16:687-700

39. Gormaz JG, Rodrigo R, Videla LA, Beems M. Biosynthesis and bioavailability of long-chain polyunsaturated fatty acids in non-alcoholic fatty liver disease. Prog Lipid Res. 2010;49:407-19.

40. Sharma L, Kaur J, Rishi P, Shukla G. Plasmodium berghei: influence of infection on the oxidant and antioxidants levels in pregnant BALB/c mice. Exp Parasitol. 2012;131:215-22.

41. Poli G. Pathogenesis of liver fibrosis: role of oxidative stress. Mol Aspects Med. 2000;21:49-98.

42. Caro AA, Cederbaum Al. Oxidative stress, toxicology, and pharmacology of CYP2E1. Annu Rev Pharmacol Toxicol. 2004;44:27-42.

43. Van den Steen PE, Van Aelst I, Starckx S, Maskos K, Opdenakker G, Pagenstecher A. Matrix metalloproteinases, tissue inhibitors of MMPs and TACE in experimental cerebral malaria. Lab Invest. 2006;86:873-88.

44. Prato M, Giribaldi G, Polimeni M, Gallo V, Arese P. Phagocytosis of hemozoin enhances matrix metalloproteinase-9 activity and TNF-alpha production in human monocytes: role of matrix metalloproteinases in the pathogenesis of falciparum malaria. J Immunol. 2005;175:6436-42. 